\title{
Infrastructure of an Agile Supply Chain System: A Virtual Agent Modeling Approach
}

\author{
H.C.W. Lau and Christina W.Y. Wong \\ Dept. of Industrial \& Systems Engineering \\ The Hong Kong Polytechnic University, Hong Kong
}

\begin{abstract}
This paper attempts to suggest an infrastructural framework for the design and development of an agile supply chain system which is characterized by its ability to cope with unprecedented changes related to the management of suppliers and the flow of parts within the value chain of the entire production network. In particular, a virtual agent modeling approach, which provides the adaptive and predictive capability to meet the requirements of this agile system, is proposed. The infrastructure of this agile supply chain system highlights the deployment of virtual agents, which are basically intelligent functional objects emulating the behavior of human beings with relatively high adaptability. The significance of contribution of this paper is concerned with the suggestion of an infrastructural framework, which is able to enhance the agility as well as adaptability of value-added activities in a supply chain, thereby providing more alternatives and ideas for those researchers who are interested in this field of study.
\end{abstract}

\section{Introduction}

In general, supply chain is a set of activities that cover enterprise functions from ordering and receiving of raw materials through the manufacturing of products in relation to the distribution and delivery to the customers. By flattening out the organizations and making use of "ad-hoc" workgroups or project teams, customer-focused business activities can be more responsive to ever-changing market conditions. To meet the relatively unpredictable customer demands, the agility of the supply chain is an important issue that is justifiable to be addressed. In general, agility is the ability of an enterprise to adapt to unforeseeable changes and this is different from flexibility which is more concerned with the ability of companies to respond to a variety of customer requirements that exist within the defined constraints [2]. Apart from the issue on customer requirements, the study on information flow within the supply chain entities is equally important in order to implement an effective network that is based on the formation of a value chain consisting of functional entities, providing timely and accurate information to achieve the efficient management of suppliers as well as the flow of parts.

From another point of view, there are activities taken place within a supply chain and this movement of activities is generally referred to as the workflow which encompasses various types of tasks that can be performed in one time interval. Evidences $[18,23]$ have shown that the adoption of various workflow approaches can help regu 
late and monitor the flow of information around the company. In general, in order to stay responsive towards market condition, both flexibility and agility are the important factors that need to be given appropriate consideration in the formulation of a company workflow $[6,13]$. As agility is more related to the "coping with the unpredictables", it is of utmost importance in a marketplace with growing uncertainty. The development of a supply chain system with such agility is the subject of study of this research.

The requirement of the capability of agility lends itself to the adoption of virtual agent modeling approach which focuses on the deployment of virtual agents composed of software programs designed to accomplish specific tasks like "real" human agents possessing specialized skills. With virtual agents that may equip themselves with certain level of intelligence, agility of the supply chain workflow can be enhanced. However, the technique to include the element of intelligence to the whole workflow needs to be carefully studied. In general, the virtual agent model is characterized by its communication between a set of autonomous, collaborative and independent agents which are designed to be business-oriented and try to accomplish business goals in a complex and ever-changing market $[29,22]$. In general, the technologies incorporated into the virtual agent model are mostly concerned with object technology, rule-based reasoning and even generic algorithm, all of which are not developed for speculating upcoming events. In this respect, it seems necessary that a certain "ingredient" of predictability such as projection of possible outcomes needs to be incorporated in order to enhance the "intelligence" of the agents.

The underlying technology of this agile supply chain system is the synergetic combination of neural networks and object technology, both of which are to be adopted to leverage the level of agility of the system. Neural networks, also called learning automata, are capable to learn relationships among complex sets of data through a learning process whereas object technology is featured by its encapsulation of both data and methods (functions) within a data object. The blend of these two technologies provide the perfect ingredient for the formation of virtual agents which are basically "intelligent functional objects" emulating the behavior of human beings albeit in a relatively adaptive way. These virtual agents will act as the "knowledge worker" of this supply chain, facilitating the efficient workflow of the entire supply chain network. With the presence of these agents, various issues such as process control, customer classification, business partner selection and demand prediction can be carried out in a way based on some adaptive reasoning mechanism. An infrastructural framework of an agile supply chain, capitalizing on the latest development in various emerging technologies, is proposed. This research is expected to have significant impact on the future development of supply chain workflow by virtue of its capabilities to progressively introduce machine self-learning and intelligence to the whole operating supply chain network over time.

\section{Paradigms of Supply Chain Workflow}

Recent years have seen significant changes made in terms of enterprise strategy and manufacturing paradigms particularly for those companies which are keen to remain world competitive in this ever-changing and volatile market $[3,14,31]$. As the manu- 
facturing market is becoming more borderless by national borders, a number of global supply chain networks have been established, taking advantage of the fast-growing networking and information technologies [27]. Study indicates that while a number of infrastructural frameworks related to dispersed supply chain systems have been described in contemporary publications [14, 21, 30, 31], the detailed structure and formulation of a "smart" monitoring infrastructure for such a system has not received too much attention.

Review on contemporary publications indicates that whilst the research findings so far have contributed to the progressive introduction of intelligence level to the models in terms of tasks decomposition and optimization of process planning operations, the machine self-learning aspect and in particular, the ability to predict possible outcomes, has not achieved the anticipated research advances $[4,7,11,12,25,28$, 30]. Machine self-learning enables the progressive addition of intelligence and corporate information to a system through a systematic knowledge creation process. This is automatic and self-tuned, thereby continually adding value to the involved system in terms of creation of a smart information management system for the monitoring of a dispersed manufacturing network. The reason of deficiency of this machine learning feature in the previous model is clear. Rule-based reasoning works by narrowing attention to a specific problem domain, then codifying human-developed rules of thumb and patterns of reasoning and the recommendations provided are normally similar to what a human expert would conclude given the same evidence whereas object technology is featured by its inheritance of data to related objects and its "properties plus behaviours" encapsulation. However, research findings based on the past years of study $[16,20,30]$ show that the self-learning feature although exists yet is not remarkable due to the distinct inherent features of these technologies which are not specifically designed for the building of an autonomous knowledge creation mechanism with the ability to predict events - should they occur at a later stage.

This paper presents an agile supply chain workflow which incorporates a combination of technologies and can be deployed in a network of companies for converting complex business data into smart information, detecting opportunities and suggesting business strategies. The main feature of such a system is concerned with its selflearning capability which is favourable to the progressive introduction of intelligence and useful knowledge to the system database over time.

\section{Tools and Technologies}

There are various technologies and relevant tools which can be used for the development of a high performance supply chain system as described in the following context.

\subsection{Distributed Object Technology}

The efficiency of the supply chain is strongly related to the efficiency and accuracy of information exchange among the business partners and the frequency of information updating within the whole network [1]. With the emergence of the need to do business globally, it is crucial that information from various partners is able to be interchanged 
in a seamless, timely and accurate manner. However, the reality is that individual business partners are likely to be using dissimilar computing platforms with different formats and the efficient exchange of information is undoubtedly a concern that needs to be addressed.

To build a supply chain system as interoperable as possible, distributed object technology can provide the support of building a cross-platform information exchange network. The approach of deploying the Object Request Broker (ORB), which is built upon the distributed object technology, can be considered to increase the degree of integration and provide more useful features of the proposed supply chain system. The ORB can be regarded as a common interface over which requests are passed between objects (data), thereby ensuring interoperability between applications on different machines in a heterogeneous distributed environment. Common Object Request Broker Architecture (CORBA), which is built around an ORB, can be regarded as a standard architecture for distributing objects in networks, aiming to simplify the communication in a heterogeneous environment.

In general, the CORBA standard provides flexibility on the company's investment (to make the right choice for its particular environment and needs), improve the manageability (more effective information handling on business activities and relationships) and enhance the speed on application development (better communication between functional departments).

The need of a common standard in facilitating the realization of a cross-platform "information object" has prompted Microsoft to develop its DCOM (Distributed Component Object Model) architecture, based on which services to build distributed applications can be developed within the Windows environment. The detailed architecture of DCOM is covered in a number of articles and publications [5] and not to be covered here. In short, DCOM is an architecture that enables components (processes) to communicate across a network which may contain heterogeneous platforms, thereby providing services such as distributed messaging, object request brokering, distributed transaction services, and data connectivity services - over its own Remote Processing Control (RPC) mechanism [26].

In general, the benefits of using DCOM include (i) ability to enable a robust transaction processing based on Windows NT platform, (ii) virtually free to be bundled with Windows NT. Generally speaking, DCOM services are very closely linked with the Windows NT platform. In order to promote DCOM as a cross-platform product, Microsoft has broadened the support of DCOM to Unix with the collaboration with third-party companies. However, there is certain degree of limitation due to the inherent differences of various operating systems.

The purpose of using distributed object technology (CORBA and DCOM) is to ensure interoperability between applications on different machines in a heterogeneous distributed environment. This technology can simplify the communication between the heterogeneous objects and each business objects of the supply chain can remain unique with shared data and logic elements. This approach is able to improve the manageability of the company (more effective information handling on business activities and relationships) and enhance the speed on application development via better communication between functional departments. In brief, distributed object technology is characterized by its provision of a transparent information communication platform. This feature allows a wide range of organizations within the supply chain to 
have transparent access to information and data. In effect, the boundaries between applications disappear and each object in an enterprise-wide environment can locate any other object without having to know where the object is located. Once this distributed platform is established, a company can change any one application without having to worry that any other application will be affected. This feature is useful in a supply chain network where companies from various regions need to share information in a timely manner without worrying about the platforms they are with.

\subsection{Neural Networks}

The neural network serves to provide the recommended change of parameters based on what the network has been trained on. As such, it is important that enough data sample for the input and output layers are provided for training purpose. Neural network is a technology that has typically been used for prediction, clustering, classification and alerting of abnormal pattern. Parroting the operation of the human brain, neural network technology comprises many simple processing units connected by adaptive weights. They create predictive networks by considering a "training set" of actual records. In theory, the formation of neural network is similar to the formation of neural pathways in the brain as a task is practiced. Also a neural network refines its network with each new input it considers. To predict a future scenario, the neural network technology is able to work with a training set of data from the past history of records. It will use the training set to build a network, based on which the neural network is able to predict future scenario by supplying a set of attributes. As neural networks are meant to learn relationships between data sets by simply having sample data represented to their input and output layers, the training of the network with the layers mapped to relevant parameter values with the purpose to develop the correlation between these two groups of data will not, in principle, contradict the basic principle of neural network.

\subsection{Rule-Based Reasoning}

In general, rule-based reasoning works by narrowing attention to a specific problem domain, then codifying human-developed rules of thumb and patterns of reasoning, providing recommendations that are normally similar to what a human expert would conclude. Regarding the design of Rule-Based Reasoning (RBR) mechanisms, it can be seen that a number of contemporary publications in this area are available $[10,15$, 19, 24]. Krishnamoorthy and Rajeev [15] describe in detail the operations of two main methods of inference or RBR, namely forward and backward chaining. Backward chaining is a goal-driven process, whereas forward chaining is data driven [9]. In order to illustrate the operation of the inference mechanism, Krishnamoorthy and Rajeev [15] describes a practical example for the selection of bearings with 16 rules and a complex "knowledge net" included The structure and the coding of the rules are explained in detail in the example. In another article, a Knowledge-Based Front End (KBFE) expert system has been developed to circumvent the restriction of knowledge-representation formalisms, including frames, classes, objects and list [10]. This KBFE system is characterized by the flexibility of its inference technique. Forward chaining is initially performed on base rules, and backward chaining is implemented, where appropriate, in order to prove individual antecedents of the base rules. 


\section{Neural Object Module}

The proposed infrastructure of the agile supply chain system comprises a variety of virtual agents responsible for different tasks. There are production scheduling VA, purchasing VA, resource planning VA, transportation management VA and logistics planning VA. Agents of specific skills can be deployed to the system as deemed necessary and can be removed when their existence is no longer required.

Apart from the traditional job activities such as scheduling, these agents are also capable for discovering patterns and clusters in business data and trigger processes when data or events occur that are outside normal patterns. In brief, VAs are for selflearning systems [8] and they are particularly capable to handle real-life ambiguous situations in which the best of us have learned to extrapolate probable outcomes based on the pattern of events we see and then decide on the action to take. In general VAs are equipped with two main technologies including (i) neural networks which are featured by their adaptive statistical reasoning and the distinct ability to learn with proven applications in inventory optimization, factory control, customer classification, process planning optimization as well as material demand prediction, and (ii) object technology which has three main features including encapsulation, inheritance and polymorphism [17]. The synergetic combination of these two technologies is named Neural Object Technology (NOT) in this research.

With the introduction of the NOT, a Neural Object Module (NOM) can be formulated. NOM, which comprises a number of neural agents responsible for undertaking various tasks, includes a rule-based reasoning mechanism for tasks decomposition and allocation to various neural agents. The proposed NOM links with the existing business objects of the supply chain network. When confronted with a job request, the rule-based reasoning mechanism undergoes an inference process and deduces the associated solutions, resulting from the process of decomposition and allocation of tasks based on available information. The coordination and monitoring of the progress of the tasks are undertaken by appropriate VAs. As VAs possess the synergetic features of both neural networks and object technology, they are specialized in predicting probable outcomes by learning from the past experience and data (neural networks features) as well as encompassing their own attributes and methods (object technology features), all of which are essential for the operations that emulate the "smart" behaviour of human operators. In general, this NOM will provide critical business suggestions for running the whole supply chain network. In short, this NOM can be deployed on an existing operational network with minimal disruption in order to improve the performance and functionality of the running dispersed network and in particular, it greatly enhances the central-management role of the manufacturing network.

\section{Infrastructure of an Agile Supply Chain System}

The NOM is the "brain" of the whole agile supply chain system. However, apart from this monitoring and control module, there are three others to form the whole framework, including Outside Interface (OI), Business Objects (BO) and System Reposi- 
tory (SR). The OI module is meant to handle two main activities including (i) job request for capturing data of request from external sources and (ii) conversion of external data to match the format of rule-based reasoning mechanism which normally needs some input data as facts to trigger the "firing" of rules. The BO module comprises a number of business objects which contain relevant group of related data used in business processes such as document processing, task allocation and workflow planning. Different companies may have various business natures and subsequently the relevant activities are also dissimilar. In this respect, various business objects should be built in accordance with the related nature of work. Business object are created based on the underlying principle of object technology which has been discussed in the previous context. To ensure the exchange of data within various data objects, a distributed mechanism is devised in the proposed infrastructure, adopting the Distributed Component Object Model (DCOM) architecture which allows data communication across a network in a distributed way. DCOM provides distributed messaging services, object request broker services, distributed transaction services, and data connectivity services [26] and through the support of the DCOM architecture, information from business objects can be exchanged within the supply chain suppliers in a distributed way.

The information flow among the virtual agents and the various Information Systems (IS) in supplier chain partners. Basically the information flow can be categorized into two levels, i.e. strategic and operation. In the strategic level, various virtual agents such as order acquisition VA, scheduling VA, transportation management VA, resource management VA and logistics VA work together to share the required data and compute the needed data objects to the partner companies. The technique to be recommended is based on the deployment of a workflow VA for keeping the needed information. Data interchanges between the VAs and system repository are via ORB, in order to enable efficient data exchange among various information systems from business partners that may reside in distributed platforms over geographically-isolated regions. These agents are created based on corresponding functions performed in traditional supply chain. Concurrently, they aim to develop preliminary business activity plans and scheduling strategies, which are then sent to the transaction VA at the operational level.

Each transaction agent makes particular plans and scheduling strategies based on its resource capacity information that is collected from business partners. Then each transaction agent sends the corresponding task to the business partners. At the same time, information systems in the business partners interact with the system repository for updating information. Thus, the workflow agent executes the particular plans and scheduling strategies that are received from each transaction agent. It should be noted that the strategic level is more concerned with the information flow among the web server platform, the data format of which may not be fully compatible with the database platform adopted by individual partner companies of the operation level handled by the transaction VA. In this respect, a Data Communication Translator (DCT) is adopted to ensure the smooth data transfer between the strategic level and the operation level. 


\section{$6 \quad$ Testing and Evaluation}

Based on the proposed system framework, a prototype system has been under development. A testing and evaluation plan, which aims to determine the feasibility of such system in a real industrial situation, has been scheduled into three phases as shown in the following.

1. The first phase involves the technical evaluation of the design and operations of the system prototype, by verifying that the object-oriented routines are doing the job as specified in the system framework.

2. The second phase is concerned with system evaluation. The prototype, with modifications made according to the problems encountered in the first phase, is deployed in a local area network, and the results are observed and recorded by the project team members.

3. The third phase is concerned with the site evaluation of the system. It is important that the agile supply chain system is to be field-tested by the real end-users, in order to determine the possible problems when operating in a practical environment.

At the present stage, the first phase has been completed, and phase two will commence once the modification of the system prototype and the setting up of a local area network have been finalized.

\section{Conclusion}

In order to attain business in the information age, companies are required to integrate business activities into a global response strategy. To cope with unpredictable changes, companies need to conduct their business activities in a flexible manner, and this task cannot be achieved without the deployment of an agile supply chain system. This paper suggests an infrastructural framework, involving various emerging technologies, for the design and development of an agent-based supply chain system with the distinct feature of the ability to cope with unexpected changes with the support of a machining learning mechanism. Further research on the infrastructural framework particularly relating to the synergetic combination of the two technologies (object technology and neural network) is needed in order to leverage the "intelligence" level of the virtual agents. In general, the proposed infrastructure paves the way for an approach to achieve agility of a supply chain system using a combination of tools and techniques. The result, if handled effectively, is essential to provide insights related to the strategic use of the supply chain concept, which can enhance company's competitiveness in a significant way.

\section{References}

1. ADC News \& Solutions (magazine articles) (1998), "Driving the supply chain with better information", ADC News \& Solutions online, News and Application Trends, May, 1998, http: / / www. manufacturing . net/magazine/adc / 
2. Backhouse, CJ \& Burns, ND (1999), "Agile value chains for manufacturing - implications for performance measures", International Journal of Agile Management Systems, Vol. 1, No. 2, 76-82.

3. Bidanda, B., Cleland, D.I., and Dharwadkar, S.R. (1993). Shared Manufacturing: A Global Perspective, McGrawHill, pp. 2-9, 15-16.

4. Bose, R. (1996), "Intelligent agents framework for developing knowledge-based decision support systems for collaborative organizational processes", Expert Systems with Applications, 11, 247-261.

5. Eddon, G., and Eddon, H. (1998), Inside distributed COM, Microsoft Press, April.

6. Ellis, S., Keddara, K. \& Rozenberg, G. (1995), "Dynamic Change within Workflow Systems", ACM Conference on Organizational Computing Systems (COOCS 95).

7. Etzioni, O. and Weld, D.S. (1995) "Intelligent agents on the internet: Fact, fiction, and forecast", IEEE Expert, 10, 44-50.

8. Eunice, J. (1997), "Neural networks ninety nine", Illuminata, Nashua (http: / / www.illuminata.com)

9. Giarratano, J.C. and Riley, G.D. (1993) Expert Systems: Principles and Programming, International Thompson Publishing.

10. Hartle, S.L. and Jambunathan, K. (1996), "Knowledge Representation and Inferencing Techniques Developed for a Knowledge-based Front End", Engineering Applications of Artificial Intelligence, Vol. 9, No. 3, 245-259.

11. Hedberg, S.R. (1995), "Intelligent agents: The first harvest of softbots looks promising", IEEE Expert, 10, 6-9.

12. Jennings, N.R. and Wooldrige, M. (1995), "Intelligent agents: Theory and practice", The Knowledge Engineering Review, 10.

13. Kappel G., Lang P., Rausch-Schott S. and Retschitzegger W. (1995), "Workflow Management Based on Object, Rules and Roles", IEEE Data Engineering, March.

14. Karlsson, C. and Ahlstrom, P. (1997), "A lean and global smaller firm", International Journal of Operations and Production Management, Vol. 17, No. 10, pp. 940-952.

15. Krishnamoorthy, C.S. and Rajeev, S. (1996) Artificial intelligence and expert systems for engineers, CRC Press.

16. Lau, H., Tso, SK and Ho, J. (1998), "Development of An Intelligent Task Management System in a Manufacturing Information Network", Expert System with Applications, Vol. 15 , pp. $165-179$.

17. Lau, H., Wong, T.T. and Pun, F. (1999), "Neural-fuzzy modelling of plastic injection molding machine for intelligent control", Expert Systems with Applications, Vol. 17, pp. 33-43

18. Lawrence, P. (Editor) (1997), Workflow handbook 1997, John Wiley \& Son, Chichester.

19. Lee, J.K. and Kwon, S.B. (1995). "ES: An Expert System Development Planner Using a Constraint and Rule-Based Approach”, Expert Systems with Applications, Vol. 9, No. 1, 314.

20. Lee, W.B and Lau, H. (1999), "Multi-agent modeling of dispersed manufacturing network", Expert System with Applications, Vol. 16, No. 3, pp. 297-306.

21. Link, D., Darlow, N.R. and Baines, T.S. (1997), "A framework for the role of manufacturing strategy in global manufacturing", Proceedings of CIRP International Symposium Advanced Design and Manufacture in the Global Manufacturing Era, pp. 417-422. Hong Kong.

22. Nwana, H. (1996), "Software Agents: An Overview", Knowledge Engineering Review, 11(3), pp. 205-244, October/November.

23. Poyssick, G. \& Hannaford, S. (1996), Workflow Reengineering, Adobe Press, Mountain View, California. 
24. Ragothaman, S., Carpenter, J., and Buttars, T. (1995). "Using Rule Induction for Knowledge Acquisition: An Expert Systems Approach to Evaluating Material Errors and Irregularities", Expert Systems with Applications, Vol. 9, No. 4, 483-490.

25. Ramsus, D.W. (1995) "Intelligent agents: PC", AI, 9, 27-32.

26. Rosemary, R. (1998), DCOM Explained, Digital Press, Boston.

27. Shi, Y., Gregory, M.J. and Naylor, M. (1997), "International manufacturing configurations map: a self assessment tool of international manufacturing capabilities", Integrated Manufacturing Systems, 8/5, pp. 273-282.

28. Sinha, A.P. and Popken, D. (1996), "Completeness and consistency checking of system requirements: An expert system approach", Expert Systems with Applications, 11, 263273.

29. Sycara K., Decker, K., Pannu, A., Williamson, M. and Zeng, D. (1996), “Distributed Intelligent Agents", IEEE Expert, December.

30. Tso, SK, Lau, H., Ho, John \& Zhang, WJ (1999), "A Framework for Developing Agentbased Collaborative Service Support System in a Manufacturing Information Network", Engineering Applications of Artificial Intelligence, Vol 12, pp. 43-57.

31. Vastag, G, Kasarda, J.D. and Bonne, T. (1994), "Logistical support for manufacturing agility in global markets", International Journal of Operations and Production Management, Vol. 14, No. 11, pp. 73-85. 